\title{
Acceso a medicamentos para estudiantes que asisten al área de salud de la VOAE en ciudad universitaria de la UNAH
}

Eleana Lizette Varela Blanco ${ }^{1}$

Nilda Suyapa Barahona ${ }^{2}$

\section{RESUMEN}

La Universidad Nacional Autónoma de Honduras (UNAH), a través de la Vicerrectoría de Orientación y Asuntos Estudiantiles (VOAE), ofrece a los estudiantes universitarios los servicios de bienestar estudiantil con diferentes áreas de atención para mantener una calidad de vida sana y disminuir el estrés. La investigación se enfocó en la población estudiantil que asiste al área de salud de ciudad universitaria, en busca de atención médica. Se encontró en el informe del área de salud y en la investigación realizada, que más del treinta y cinco por ciento (35\%) de la población total utiliza los siguientes servicios: consulta médica, odontológica, psicológica, análisis clínico y farmacia. Debido a los hallazgos encontrados, se propone la creación de un "Programa de educación en medicina preventiva en ciudad universitaria”, el cual consistirá en la implementación de una unidad ejecutora de todas las acciones coordinadas por el área de salud de la VOAE y las unidades académicas del área de salud involucradas y definidas para llevar a cabo actividades de formación y capacitación en materia de medicina preventiva, con el fin de educar para la prevención en salud oral, física, mental y vulnerabilidad psíquica. Lo anterior se logrará realizando conferencias, charlas y diferentes talleres de capacitación de manera continua y de ser posible con obligatoriedad, para incidir en la disminución de la medicina curativa del cuadro básico de enfermedades de ciudad universitaria, procurando reducir la cuenta presupuestaria institucional en la prescripción de medicamentos.

Palabras clave: atención primaria, incidencia, fármaco, vigilancia, prescripción, inaccesible

\footnotetext{
${ }^{1}$ Profesora universitaria y estudiante de Maestría de Tecnología y Control de Medicamentos, Facultad de Química y Farmacia, UNAH: eleana.varela@unah.edu.hn

${ }^{2}$ Asesora, Profesora de la Maestría de Tecnología y Control de Medicamentos, Facultad de Química y Farmacia, UNAH: nilda.barahona@unah.edu.hn
} 


\section{ABSTRACT}

La Universidad Nacional Autónoma de Honduras (National Autonomous University), UNAH, offers student wellbeing services, with different attention foci, aiming to maintain a healthy lifestyle and diminished stress. Such services are provided through the Vicerrectoría de Orientación y Asuntos Estudiantiles (Guidance and Student Affairs Rectory), VOAE. Student population who attended the university's medical center was the research's focus. The health center's report apprised that more than $35 \%$ of the population sought aid in the following areas: consultation, odontology, psychology, clinical analysis, and pharmacy. Given these findings, a Preventive Medicine Educational Program (for Tegucigalpa's campus only) was proposed. The program consists of both VOAE's health department plus Health Faculty Academic Unit's actions and activities, which will serve as an executing unit. Such will carry out training activities oriented towards preventive medicine, specifically: oral health, physical health, mental health, and psychic vulnerability. Talks, conferences, and training workshops will be held continually and if necessary, made obligatory to attend. The program aims to decrease common illnesses' incidence, thus decreasing institutional budgetary occurrence in prescribing drugs.

Keywords: primary attention, incidence, pharma covigilance, prescription, inaccessible 


\section{INTRODUCCIÓN}

El presente estudio nace como una inquietud relacionada con los servicios que ofrece el área de salud de la VOAE, en especial resaltando las prescripciones de medicamentos, el alto precio de estos y las asignaciones presupuestarias fijas que inciden sobre una adecuada atención para el usuario de los servicios médicos en la ciudad universitaria de Tegucigalpa.

También tiene como finalidad evaluar los requerimientos de medicamentos, la cobertura parcial o total de las prescripciones médicas, para sugerir una vía que contribuya al mejoramiento de la calidad de atención en salud y la identificación de enfermedades más frecuentes.

El proceso de investigación sirvió de apoyo para demostrar la necesidad de modificar el enfoque de atención médica que se emplea en la actualidad, mediante un programa de educación en medicina preventiva en la ciudad universitaria y explicar con precisión las tendencias de crecimiento de la población universitaria, el incremento futuro de la demanda de medicamentos que ofrece el servicio de salud y detectar la necesidad de realizar programas y proyectos interdisciplinarios.

El hablar de Universidades como promotoras de espacios de salud integral va acorde a, entre otros aspectos, a hablar de la reorientación de los servicios médicos universitarios hacia el fomento de estilos de vida y entornos saludables, priorizando el componente preventivo de la enfermedad y de la promoción de salud, con una perspectiva integral e integradora.

\section{METODOLOGÍA}

Se observaron los fenómenos como se dieron en su contexto natural y posterior análisis, por lo que utilizó el tipo de investigación no experimental con un diseño transeccional exploratorio, ya que se recolectaron datos en el segundo periodo académico de 2011 y actualizada la información en el segundo periodo académico de 2014, para hacer inferencias con respecto al cambio, sus determinantes y consecuencias, con el propósito de conocer las necesidades de la población objeto de estudio. Dicha población estuvo conformada por los estudiantes de las diferentes facultades de la ciudad universitaria en Tegucigalpa, que asisten al área de salud, con información actualiza- 
da al segundo periodo de 2014. En el tamaño de la muestra se utilizó un enfoque cualitativo para la toma de la muestra debido a que se consideró la población que asiste al área de salud, concluyendo en un número de cincuenta estudiantes.

Se utilizaron dos métodos: el método probabilístico estratificado, ya que la muestra se tomó de las diferentes facultades de la UNAH y el método no probabilístico, debido a la selección de la unidad de muestreo. Se usó el método por conveniencia, ya que el número de elementos que van a integrar la muestra se selecciona de una manera aleatoria (Jany, 2009).

Se aplicaron diferentes instrumentos de exploración de información, tales como encuestas a los estudiantes, encuesta y entrevista al personal médico. Otra fuente de información es el informe anual que presentó el área de salud a la VOAE (2011), en el cual se encuentra gran parte de la información utilizada para la presente investigación.

La información obtenida en las encuestas y entrevistas se procesó con el programa estadístico EXEL. Para llevar a cabo el procesamiento de los mismos, se empleó la distribución de frecuencias con el fin de obtener el conteo del número de repuestas asociadas con los valores de cada variable, cuyos resultados fueron expresados en términos numéricos y porcentuales. Esta información validó la obtenida en el informe anual del área de salud.

La Organización Mundial de la Salud (2011), estima que más de un tercio de la población mundial no tiene garantizado el acceso a los medicamentos esenciales y existen diversas razones para esa falta de acceso. En el mundo entero, la razón principal es la dificultad financiera (los medicamentos tienen un costo que excede la capacidad de los consumidores medios), pero los factores jurídicos, infraestructurales, culturales y de distribución, son también graves obstáculos. La influencia de cada uno de esos factores varía de un país a otro, así como la frecuencia de las enfermedades.

En este abordaje se consideran los medicamentos un insumo terapéutico importante (pero no el único). Este concepto se basa en el desarrollo de los servicios farmacéuticos, distinto del foco actual en los productos y su consumo. La iniciativa sobre servicios farmacéuticos (SF) basados en atención primaria de salud (APS) comenzó a finales de 2008 (OPS/OMS, 2010).

La estrategia de fortalecimiento de los servicios farmacéuticos basados en atención 
en salud, centrados en el paciente y menos en el medicamento, es parte del marco de cooperación técnica de la Organización Panamericana de la Salud/Organización Mundial de la Salud (OPS/OMS) en la región de las Américas.

\section{ANÁLISIS DE RESULTADOS}

\section{Presupuesto del área de salud}

Para los años 2010 y 2011, el presupuesto total para el área de salud fue de L. 43, 425, 379.00 y lo destinado para productos farmacéuticos fue de L. 358, 946.99; asimismo, en el año 2012, el presupuesto total fue de L. 50, 965, 782.41 y lo destinado a productos farmacéuticos fue de L. 538,946.00. Es de resaltar que el incremento del presupuesto total fue de alrededor L. 7.5 millones para el 2012, de los cuales únicamente se asignaron L. 200, 000.00 a productos farmacéuticos. (Comisión de Transparencia, 2013)

El costo promedio aproximado por cada estudiante atendido en el área de salud, está directamente relacionado al presupuesto anual asignado y a la cantidad total de estudiantes atendidos cada año. Según el informe anual del área de salud, para los años 2012 y 2013 se asistieron un total de 17, 776 y 17, 789 estudiantes, respectivamente; lo que representa el 39 y $37 \%$ de la población total matriculada en la ciudad universitaria para esos años. En consecuencia, el costo promedio aproximado por atención sería de L. 2,867.11por estudiante.

\section{Alto precio de los medicamentos}

El mercado de productos de prescripción representa el $92 \%$ de las ventas y el mercado de productos populares o venta libre un $8 \%$. Ambos mercados están creciendo, un $15 \%$ y un $12 \%$, respectivamente. Se conoce que aproximadamente se comercializan más de 10,000 presentaciones de medicamentos en la región centroamericana (Castellanos, 2007). En la tabla 1 se muestra el incremento de precios de algunos medicamentos utilizados en el área de salud de la VOAE, información proporcionada por el Departamento de Compras de la UNAH de los años comprendidos entre el 2011 y 2013. 


\section{Tabla 1. Incremento anual de los medicamentos}

\begin{tabular}{|c|c|c|c|c|c|}
\hline \multicolumn{2}{|c|}{ Tipo medicamento } & Presentación & 2011 & 2012 & 2013 \\
\hline \multirow{3}{*}{ Antibiótico } & Amoxicilina & Capsula $500 \mathrm{mg}$ & L. 1.00 & L. 1.00 & L. 1.20 \\
\cline { 2 - 6 } & Dicloxacilina & Capsula $500 \mathrm{mg}$ & L. 2.40 & L. 2.87 & No* \\
\cline { 2 - 6 } & Trimetropin Sulfa & Tabletas $80 / 40$ & L. 0.65 & L. 0.70 & No* \\
\cline { 2 - 7 } & Gentamicina & Ampolla $160 \mathrm{mg}$ & No* & L. 3.93 & L. 7.40 \\
\hline \multirow{2}{*}{ Antiinflamatorio } & Ibuprofeno & Tabletas $400 \mathrm{mg}$ & L. 0.45 & L. 0.56 & No* \\
\cline { 2 - 7 } & Diclofenaco & Tabletas $225 \mathrm{mg}$ & L. 0.52 & L. 0.55 & L. 0.72 \\
\hline \multirow{2}{*}{ Antialérgico } & Loratadina & Tableta & L. 0.57 & L. 0.75 & L. 1.35 \\
\hline Antitusivo & Dextrometorfano & Frasco $30 \mathrm{ml}$ & L. 10.00 & L. 10.00 & L. 15.00 \\
\hline Antiácido & Ranitidina & Tableta $300 \mathrm{mg}$ & L. 1.00 & L. 1.15 & L. 1.57 \\
\hline Antiparásitos & Albendazol & Tabletas & L. 1.21 & L. 1.25 & No* \\
\hline
\end{tabular}

* No se realizaron compras de ese medicamento.

Fuente: Elaboración propia con información del Departamento de Compras de la UNAH, 2014.

De acuerdo al análisis del rubro de las medicinas del índice de precios al consumidor que dicta Honduras en cifras del Banco Central de Honduras $(\mathrm{BCH}, 2011)$ y otras fuentes de estudio como el Instituto Nacional de Estadísticas (INE, 2010), se observa una variación mensual del $4.0 \%$ del índice, el que repercute en 0.134 veces en el índice general de los precios al consumidor y los ingresos de las familias. Este comportamiento se debe al aumento de los precios de los medicamentos y otros productos farmacéuticos, esenciales para la buena salud física de los estudiantes y la población en general del país.

Según Honduras en cifras (BCH, 2011), se realiza la siguiente imagen de valoración para el porcentaje de incremento en la inflación nacional en el periodo comprendido entre los años 2007 a 2011. Como se muestra en la figura 1, cada año hay un incremento sustancioso en la inflación de los precios al consumidor en una forma general, afectando de igual manera el precio de los medicamentos. Para el 2009 se produjo un elevado porcentaje de inflación debido a problemas políticos en Honduras. En los años siguientes existió un decrecimiento que se ve reflejado en la figura 1, lo que probablemente se debió a la estabilidad política del país, a pesar de la baja inversión extranjera luego de la inestabilidad política vivida en ese año. 


\section{Gráfico 1. Porcentaje de inflación}

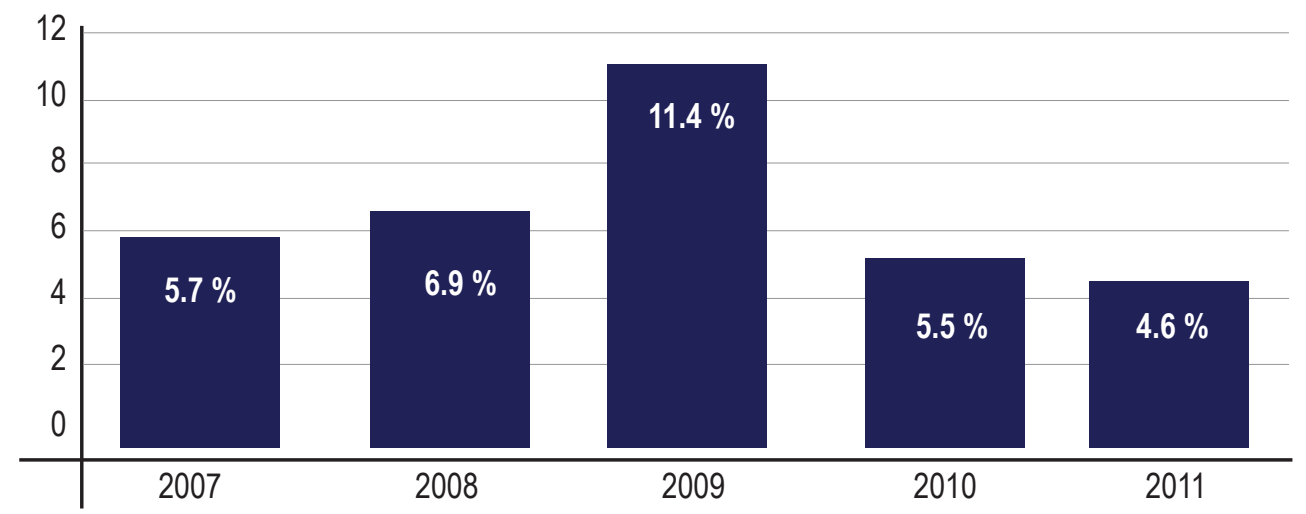

Fuente: Mundi, 2011

La inflación es un fenómeno económico que afecta a toda la población en cualquier parte del mundo; así, la población estudiantil en estudio en muchos casos está siendo afectada, ya que en su mayoría tiene un ingreso familiar igual o menor al salario mínimo. De acuerdo a la información que brinda cada estudiante que ingresa a la Universidad Nacional Autónoma de Honduras, el Sistema de Admisiones maneja una base de datos (ver figura 2).

\section{Gráfico 2. Ingreso familiar promedio mensual}



Fuente: Sistema de Admisión, UNAH, 2011 
El ingreso promedio familiar que reporta la población estudiantil, está por debajo de los diez mil lempiras, lo que evidencia la necesidad de una atención médica gratuita para los estratos sociales que perciben este ingreso, ya que en muchos de los casos sus ingresos les impiden destinar parte del mismo a los requerimientos mínimos necesarios para una salud integral.

\section{Cobertura de medicamentos}

En los años representados en la tabla 2 se evidencia el aumento progresivo de la población estudiantil y, a su vez, la demanda del servicio de salud, manteniendo una relación para los últimos dos años mayor de un treinta y cinco por ciento (35\%).

\section{Tabla 2. Porcentaje de población estudiantil asistida}

\begin{tabular}{|c|c|c|c|c|c|c|}
\hline & 2008 & 2009 & 2010 & 2011 & 2012 & 2013 \\
\hline $\begin{array}{c}\text { Estudiantes } \\
\text { matriculados }\end{array}$ & 40,696 & 40,000 & 41,455 & 42,246 & 46,205 & 47,937 \\
\hline Consulta médica & 10,373 & 10,742 & 9,979 & 11,453 & 17,776 & 17,789 \\
\hline Relación & $26 \%$ & $27 \%$ & $24 \%$ & $27 \%$ & $39 \%$ & $37 \%$ \\
\hline
\end{tabular}

Fuente: SEDI y VOAE, 2014.

Según lo expresado por la población objeto de estudio y el personal médico, por lo general se les prescribe una receta con diferentes medicamentos, la cual es cubierta de forma parcial o total dependiendo del tipo de afección que se esté presentando, es decir, a mayor complejidad menor cobertura. La tabla 3 evidencia que la cobertura de medicamentos fue de un $50 \%$ aproximadamente.

\section{Tabla 3. Porcentaje de medicamentos prescritos y entregados por paciente}

\begin{tabular}{|c|c|c|}
\hline $\begin{array}{c}\text { Cantidad de medicamentos } \\
\text { prescritos }\end{array}$ & $\begin{array}{c}\text { Porcentaje de medicamento } \\
\text { prescrito }\end{array}$ & $\begin{array}{c}\text { Porcentaje de medicamento } \\
\text { entregado }\end{array}$ \\
\hline 0 & 2.0 & 24.0 \\
\hline 1 & 26.0 & 44.0 \\
\hline 2 & 40.0 & 20.0 \\
\hline 3 & 22.0 & 10.0 \\
\hline 4 & 10.0 & 2.0 \\
\hline Total & 100.0 & 100.0 \\
\hline
\end{tabular}

Fuente: elaboración propia. 


\section{Acceso a medicamentos}

Entre menor sea el rango de ingreso familiar de la población objeto de estudio, mayor es la limitante para acceder a medicamentos fuera del beneficio que ofrece el área de salud. Se evidencia que la población estudiantil demandante de los servicios de salud, es la que tiene ingresos por debajo de los 10,000.00 lempiras; de esto se puede inferir de los resultados generales de la población, ya que la mayoría se ubica en el mismo ingreso promedio (ver figura 2).

\section{DISCUSIÓN}

Hi1: El presupuesto asignado al área de salud influye en la cobertura total de las prescripciones médicas que reciben los estudiantes, en parte, por el precio, lo cual tiene efecto en la accesibilidad de los medicamentos.

El presupuesto asignado al área de salud, en particular el de la compra de productos farmacéuticos, fue del $1.05 \%$ del total, este porcentaje representó la cantidad de L. $538,946.00$ para el año 2012. Si bien es cierto que dicha cantidad de dinero es poca, se reduce aún más por los precios de los medicamentos que aumentan progresivamente cada año.

De igual forma, cada año incrementa la población estudiantil en la ciudad universitaria y la demanda de servicios médicos integrales, sobre todo aumenta la necesidad de cobertura total de medicamentos, ya que para la recuperación de la salud estos son esenciales; esta necesidad de cobertura cada año será mayor para la población objeto de estudio, debido a que el $54 \%$ de ellos tienen ingresos económicos familiares que están por debajo de los L. 10, 000.00 (ver figura 2),consecuentemente la problemática se ve ahondada por el alto costo (ver tabla 1) y la inflación (ver figura 1). En la actualidad, la población objeto de estudio muestra un limitado acceso a los medicamentos cuando asiste al área de salud en busca de asistencia médica, ya que no reciben la totalidad de ellos, aunque hayan sido prescritos por el médico. Se observa en la tabla 3 que al $44 \%$ de la población se le entrega un medicamento de los dos que le prescribió el galeno. Es decir, que la población recibe el $50 \%$ de la cobertura de medicamentos.

El acceso a medicamentos es de vital importancia para la recuperación de la salud de la población estudiantil universitaria, ya que al no recibir la cobertura total, no posee 
un tratamiento completo, lo que conlleva a tratamientos inconclusos y a una fallida recuperación de la salud.

\section{CONCLUSIONES}

1. En el último año de la investigación se obtuvo un porcentaje de $1.05 \%$ destinado al suministro de productos farmacéuticos y medicinales, además de mantenerse fijo a través de los años; esto viene a confirmar que las asignaciones presupuestarias fijas y el incremento constante de la población estudiantil no garantizan el acceso total a los medicamentos prescritos en las consulta médica.

2. Según los hallazgos, el nivel de precio de los medicamentos constantemente está aumentando su valor, por lo que cada año se necesita mayor cantidad de presupuesto para cumplir con una dispensación apropiada.

3. El área de salud muestra un progresivo crecimiento en atención primaria en salud, debido al aumento de la población estudiantil que en cada periodo académico es mayor al anterior y un aumento en el personal de atención, con el propósito de dar respuesta a las necesidades de recuperación de la salud demandante.

\section{AGRADECIMIENTO}

A los directores de la VOAE, América Alvarado (2008-2012) y Ayax Irías (2014), por la información brindada para la presente investigación. A Luz Duarte, por su ayuda y orientación y a mis asesoras, Alba Luz Hernández y Nilda Barahona.

\section{BIBLIOGRAFÍA}

Admisión, S. D. (2011). Resumen de admisión por ingreso mensual reportado. Tegucigalpa: Resumen Informe.

Castellanos, A. (2007). Auge del mercado farmaceutico regional. Mercados \& Tendencias. Recuperado de: http://www.revistamyt.com/myt/entrevista/87-auge-del-mercado-farmaceutico-regional 
INE. (2010). Encuesta permanante de hogares de propósitos múltiples. Tegucigalpa. Mundi, I. (2011). Honduras tasa de inflación. Recuperado de: http://www.indexmundi.com/es/honduras/tasa_de_inflacion_(precios_al_consumidor).html

OPS/OMS. (2010). Servicios farmacéuticos basados en atención primaria de salud. Recuperado de: http://new.paho.org/bra/index2.php?option=com_docman\&tas$k=$ doc_view\&gid $=1019 \&$ ltemid $=614$

Organización Mundial para la Salud. ( 2011). Situacion mundial de los medicamentos 2011. Recuperado de: http://www.who.int/medicines/areas/policy/world_medicines_situation/en/index.html

VOAE. (2013). Plan estratégico VOAE julio 2013 - julio 2016. Tegucigalpa: UNAH.

VOAE. (2014). Reorganización de los servicios de salud universitarios (propuesta). Tegucigalpa: UNAH. 